\title{
Depletion of nitric oxide synthase-containing neurons in the diabetic retina: reversal by aminoguanidine
}

\author{
E. Roufail ${ }^{1,2}$, T. Soulis ${ }^{3}$, E. Boel ${ }^{4}$, M.E. Cooper $^{3}$, S. Rees ${ }^{1}$ \\ ${ }^{1}$ Department of Anatomy and Cell Biology, University of Melbourne, Parkville, Victoria, Australia \\ ${ }^{2}$ Department of Ophthalmology, University of Melbourne, Parkville, Victoria, Australia \\ ${ }^{3}$ Department of Medicine, University of Melbourne, Austin and Repatriation Medical Centre (Repatriation Campus) Banksia \\ Street, West Heidelberg, Victoria, Australia \\ ${ }^{4}$ Health Care Discovery, Novo-Nordisk, Bagsvaerd, Denmark
}

Summary A close association of neuronal nitric oxide synthase-immunoreactive (nNOS-IR) neurons with the retinal vasculature has been reported and it is proposed that activation of these neurons could be the mechanism by which retinal blood flow and metabolism are linked. Further, advanced glycation end products (AGEs) have previously been shown to be increased in the diabetic retina and aminoguanidine (AG), an inhibitor of advanced glycation, has been shown to attenuate the development of AGE accumulation as well as the progression of experimental diabetic retinopathy. This study examined the effects of short (1 and 3 weeks) and long term (32 weeks) diabetes on nNOS-containing neurons of the retina using NADPH diaphorase (NADPHd) histochemistry. In addition, the effect of aminoguanidine (an inhibitor of advanced glycation and NOS) and $\mathrm{N}^{\mathrm{G}}$-nitro-Larginine methyl ester (L-NAME) (a non-selective NOS inhibitor) on retinal nNOS-containing neurons was examined in short and long term control and diabetic rats. In a separate study, the effect of 2,3 diami- no-phenazine (NN0028) (an inhibitor of advanced glycation, but not NOS) was examined in short term (3 weeks) diabetic rats. The number of NADPHdpositive neurons per retina was reduced after one week of diabetes and remained decreased in long term diabetic rats, an effect not observed in diabetic rats rendered euglycaemic by intensified insulin treatment. Treatment of diabetic animals with aminoguanidine or NN0028 prevented the depletion in the nNOS-containing neuron number, an effect not reproduced by L-NAME. These studies suggest that the action of AG in restoring the number of nNOScontaining retinal neurons is mediated by the inhibition of AGE formation. The depletion of nNOS-containing neurons may contribute to alterations in the autoregulation of blood flow which occurs in diabetes. [Diabetologia (1998) 41: 1419-1425]

Keywords Retinopathy, advanced glycation, blood vessels, NADPH diaphorase, amacrine cells.
The pathogenesis of diabetic retinopathy has not been fully elucidated but is thought to be related to haemodynamic changes of the retinal vasculature [1]. Many vasoactive substances influence retinal hae-

Received: 23 February 1998 and in final revised form: 4 August 1998

Corresponding author: Dr. E. Roufail, Department of Anatomy and Cell Biology, University of Melbourne, Parkville, 3052, Victoria, Australia

Abbreviations: NOS, nitric oxide synthase; NADPHd, NADPH diaphorase; AGEs, advanced glycation end products; AG, aminoguanidine; L-NAME, nitro-L-arginine methyl ester; NO, nitric oxide; DR, diabetic retinopathy; NN0028, 2,3 diamino-phenazine; STZ, streptozotocin; PB, phosphate buffer. modynamics including nitric oxide (NO), a diffusible gas molecule, formed by three different isoforms of nitric oxide synthase (NOS) [2]. One isoform of NOS is neuronal NOS (nNOS) which is found throughout the nervous system and has been localised to specific cells of the retina including amacrine cells [3]. Previously we have shown that these nNOS-immunoreactive (nNOS-IR) amacrine cells lie in close proximity to the retinal vessels and that their processes can envelop the retinal vasculature [3]. This suggests that nitrergic innervation of the retinal vasculature might link retinal blood flow to metabolism [3] as has been proposed for the cerebral cortex [4].

Given the potential role for nNOS in retinal vascular control, the first aim of this study was to deter- 
mine the time course and degree of any change in the expression of nNOS in the diabetic retina, as demonstrated by NADPHdiaphorase (NADPHd) histochemistry. The present study also explored the process of advanced glycation which has been implicated in the pathogenesis of diabetic retinopathy [5]. Indeed, inhibition of advanced glycation by aminoguanidine (AG) has been shown to prevent morphological changes of diabetic retinopathy [5]. Therefore, the second aim of the study was to evaluate the effect of aminoguanidine treatment on nNOS-containing retinal neurons in short ( 3 weeks) and long term (32 weeks) streptozotocin (STZ)-induced diabetic rats. Since aminoguanidine can also act as an inhibitor of specific NOS isoforms, the effect of this agent was compared with the effect of treatment with $\mathrm{N}^{\mathrm{G}}$ nitro-L-arginine methyl ester (L-NAME), a nonselective NOS inhibitor [6]. In addition, to explore in more detail the specificity of the effect of aminoguanidine, a novel inhibitor of advanced glycation, 2,3 diamino-phenazine (NN0028), which does not inhibit NOS, was also evaluated.

\section{Materials and methods}

Animal protocol. Male Sprague Dawley rats were obtained from the Austin Hospital Biological Research Laboratory colony (Heidelberg, Australia). Diabetes was induced in rats $8-9$ weeks of age weighing 200 to $250 \mathrm{~g}$ by injection of STZ (Boehringer-Mannheim, Mannheim, Germany) into the tail vein at a dose of $55 \mathrm{mg} / \mathrm{kg}$ body weight after an overnight fast. Animals were cared for in accordance with the guidelines of the National Health and Medical Research Council (NH \& MRC, Australia) for the care of laboratory animals. All experiments were approved by the University of Melbourne Animal Ethics Committee. Animals were allowed free access to food (GR2 rat cubes, Clark King and Co., Melbourne, Australia) and water and were on a 12-h light-dark cycle.

Animals were divided into six groups: untreated control group ( 1 week, $n=6 ; 3$ weeks, $n=10 ; 32$ weeks; $n=7$ ) and diabetic groups ( 1 week, $n=6$; 3 weeks, $n=14$; 32 weeks, $n=7$ ), aminoguanidine-treated control group (3 weeks, $n=5$; 32 weeks, $n=5$ ) and diabetic groups (3 weeks, $n=10$; 32 weeks, $n=10)$ and L-NAME treated control group ( 3 weeks, $n=6$ ) and diabetic groups ( 3 weeks, $n=6$; 32 weeks, $n=6)$. In addition, one group of animals $(n=6)$ was made euglycaemic by insertion of 1.5 silastic pellets of insulin [7] after STZ injection to ensure that the effects seen were due to hyperglycaemia per se rather than to STZ toxicity. These rats were killed 3 weeks after the injection of STZ. An additional group of 3-week untreated diabetic rats $(n=4)$ were used in a study to co-localize NADPHd and nNOS. AG-treated control rats were given drinking water containing $2 \mathrm{~g} / \mathrm{l} \mathrm{AG}$ hydrogen carbonate and diabetic-treated rats were given water containing $1 \mathrm{~g} / \mathrm{l}$ of $\mathrm{AG}$ hydrogen carbonate (Fluka Chemica, Buchs, Switzerland) [8]. The NOS inhibitor L-NAME was given at the non-pressoric dose of $5 \mathrm{mg} / \mathrm{l}$ in drinking water to both control and diabetic groups for 3 or 32 weeks. Long term diabetic rats (32 weeks) received 2 units of ultralente insulin (Ultratard HM, Novo Industries, Bagsvaerd, Denmark) subcutaneously every second day in order to maintain body weight and to improve survival over the 32-week study period. Control and diabetic animals from each group were killed at 1, 3 and 32 weeks.
In the second study, the effects of 2,3 diamino-phenazine (NN0028)(NOVO, Nordisk, Bagsvaerd, Denmark) were explored. NN0028 has been shown to inhibit AGE accumulation in vitro and does not inhibit NOS (Boel et. al., unpublished data). Three groups of animals were used; control $(n=6)$, diabetic $(n=6)$ and NN0028-treated diabetic animals $(n=5)$. Diabetes was induced as above and animals in the treatment group were given $10 \mathrm{mg}$ of NN0028 per kg of body weight per day in drinking water for the three-week duration of the study.

Serum glucose concentrations were measured by the glucose oxidase technique [9] before killing the animals. Body weights and blood pressure assessed by tail cuff plethysmography [10], were measured before killing the short term diabetic rats and every 8 weeks in the long term diabetic groups. At the same time, blood was collected for measurement of glycated haemoglobin by a high performance liquid chromatography assay (Bio-rad, Richmond, Calif., USA).

Tissue preparation. Rats were killed with an intravenous overdose of sodium pentobarbital $(130 \mathrm{mg} / \mathrm{kg})$. Each animal's right eye was removed and the cornea perforated. The eyes were placed in $4 \%$ paraformaldehyde in $0.1 \mathrm{~mol} / \mathrm{l}$ phosphate buffer (PB) $\left(\mathrm{pH} \mathrm{7.4)}\right.$ for $1 \mathrm{~h}$ at $4^{\circ} \mathrm{C}$. The retina was dissected in $0.1 \mathrm{~mol} / 1 \mathrm{~PB}(\mathrm{pH} 7.4$ ) and placed in $4 \%$ paraformaldehyde for a further $2 \mathrm{~h}$ and prepared for NADPHd histochemistry.

NADPH diaphorase histochemistry. This method was carried out as described previously [3]. In brief, dissected retinas were washed $3 \times 10 \mathrm{~min}$ in Tris Buffer $(\mathrm{pH} 7.6)$. They were then left overnight in $0.2 \%$ Triton X-100 in Tris buffer (pH 7.6). Retinal tissue was then reacted for NADPHd at $37^{\circ} \mathrm{C}$ for $2 \mathrm{~h}$. Retinas were washed $3 \times 10 \mathrm{~min}$ in Tris buffer ( $\mathrm{pH}$ 7.6). They were left overnight in $0.2 \%$ Triton $\mathrm{X}-100$ in Tris buffer (pH 7.6) and then reacted for NADPHd using $0.25 \mathrm{mg} / \mathrm{ml}$ nitroblue tetrazolium, $1 \mathrm{mg} / \mathrm{ml} \beta$-NADPH, 0.5 per cent Triton X-100 in $0.1 \mathrm{~mol} / 1$ Tris buffer (pH 7.6) at $370^{\circ} \mathrm{C}$ for $2 \mathrm{~h}$. Retinas were then mounted on $1 \%$ gelatinized slides, compressed for $20 \mathrm{~min}$ and coverslipped with glycerol jelly.

$n N O S-I R$ and NADPH diaphorase double staining in diabetic rat retina. Retinal tissue from 3 -week untreated diabetic rats $(n=4)$ was first reacted for NADPHd histochemistry, (as described above), for $45 \mathrm{~min}$ at $37^{\circ} \mathrm{C}$. The tissue was then placed in $30 \%$ sucrose solution overnight. Frozen, $12 \mu \mathrm{m}$ transverse sections were cut on a cryostat and collected on $1 \%$ chromealum-coated slides and allowed to air dry for $1 \mathrm{~h}$. Sections were covered with $10 \%$ NGS in $0.1 \mathrm{~mol} / 1 \mathrm{~PB}(\mathrm{pH} \mathrm{7.4)}$ ) for $30 \mathrm{~min}$ and then incubated at room temperature in rabbit antiserum against nNOS (Auspep, Melbourne, Australia) at a dilution of 1:200 in PB containing $0.25 \%$ Triton X-100 overnight. The sections were then washed with $\mathrm{PB}(3 \times 10 \mathrm{~min})$ and then incubated in fluorescein isothocyanate (FITC) (Wellcome, Dartford, England) labelled anti-rabbit antibody for $2 \mathrm{~h}$. Sections were then washed and coverslipped with PB. A selection of $70 \mathrm{nNOS}$-IR cells was done at random and by alternating the microscope light source from incandescent to fluorescent illumination it was possible to determine whether the same cells stained positively for NADPHd and nNOS-IR.

Control experiments. Control experiments for nNOS-IR on flat mount preparations of retina included the omission of primary antiserum and its replacement with $0.1 \mathrm{~mol} / \mathrm{l} \mathrm{PB}$. Preadsorption controls were also carried out where $20 \mu \mathrm{g}$ of the peptide used as the immunogen to raise the original antiserum was added to the working dilution of primary nNOS antibody and incubated at $4{ }^{\circ} \mathrm{C}$ overnight. In both of these procedures immunoreactive staining did not occur. In control experiments for 
Table 1. Body weight, blood pressure and serum glucose in control, diabetic and treatment groups

\begin{tabular}{|c|c|c|c|c|c|}
\hline & $(n)$ & Body weight (g) & Blood pressure $(\mathrm{mmHg})$ & Serum glucose $(\mathrm{mmol} / \mathrm{l})$ & $\mathrm{HbA}_{1 \mathrm{c}}(\%)^{3}$ \\
\hline \multicolumn{6}{|l|}{ Study 1} \\
\hline \multicolumn{6}{|l|}{ A: 1 Week } \\
\hline Diabetic & 6 & $271 \pm 5$ & $118 \pm 3$ & $\begin{array}{l}8.1 \pm 0.4 \\
22 \pm 0.1^{1}\end{array}$ & $\begin{array}{l}1.5 \pm 0.09 \\
1.6 \pm 0.1\end{array}$ \\
\hline \multicolumn{6}{|l|}{ B: 3 Weeks } \\
\hline Control & 10 & $353 \pm 7$ & $113 \pm 4$ & $7.8 \pm 0.4$ & $1.3 \pm 0.1$ \\
\hline Diabetic & 14 & $194 \pm 8^{1}$ & $126 \pm 2^{1}$ & $32.1 \pm 1.0^{1}$ & $4.3 \pm 0.2^{1}$ \\
\hline Euglycaemic & 6 & $225 \pm 20$ & $124 \pm 3$ & $9.8 \pm 1.5^{2}$ & $2.2 \pm 0.3^{2}$ \\
\hline Control + AG & 5 & $347 \pm 21$ & $117 \pm 3$ & $6.9 \pm 1.0$ & $1.4 \pm 0.2$ \\
\hline Diabetic + AG & 10 & $177 \pm 26^{1}$ & $118 \pm 3^{2}$ & $33.2 \pm 2.2^{1}$ & $4.0 \pm 0.3^{1}$ \\
\hline Control + L-NAME & 6 & $360 \pm 19$ & $114 \pm 2$ & $8.0 \pm 0.5$ & $1.5 \pm 0.1$ \\
\hline Diabetic + L-NAME & 6 & $183 \pm 14^{1}$ & $120 \pm 2$ & $34.0 \pm 1.0^{1}$ & $4.4 \pm 0.2^{1}$ \\
\hline \multicolumn{6}{|l|}{ C: 32 Weeks } \\
\hline Control & 7 & $535 \pm 20$ & $118 \pm 3$ & $5.5 \pm 0.2$ & $2.8 \pm 0.1$ \\
\hline Diabetic & 7 & $378 \pm 20^{1}$ & $129 \pm 3^{1}$ & $26.8 \pm 1.0^{1}$ & $5.2 \pm 0.4^{1}$ \\
\hline Control + AG & 5 & $520 \pm 60$ & $121 \pm 4$ & $7.8 \pm 0.4$ & $2.5 \pm 0.4$ \\
\hline Diabetic + AG & 10 & $430 \pm 31^{1}$ & $131 \pm 2^{1}$ & $26.7 \pm 1.3^{1}$ & $5.6 \pm 0.3^{1}$ \\
\hline Diabetic + L-NAME & 6 & $348 \pm 18^{1}$ & $136 \pm 4^{1}$ & $25.4 \pm 0.9^{1}$ & $4.9 \pm 0.5^{1}$ \\
\hline \multicolumn{6}{|l|}{ Study two } \\
\hline \multirow{2}{*}{\multicolumn{6}{|c|}{$\begin{array}{l}\text { D: } 3 \text { Weeks } \\
\text { NN0028 }\end{array}$}} \\
\hline & & & & & \\
\hline Control & 6 & $337 \pm 11$ & $108 \pm 5$ & $7.4 \pm 1.0$ & $1.11 \pm 0.1$ \\
\hline Diabetic & 6 & $186 \pm 15^{1}$ & $124 \pm 3^{1}$ & $33.2 \pm 1.0^{1}$ & $3.8 \pm 0.3^{1}$ \\
\hline Diabetic + NN0028 & 5 & $165 \pm 11^{1}$ & $114 \pm 6$ & $33.1 \pm 3.0^{1}$ & $3.7 \pm 0.6^{1}$ \\
\hline $\begin{array}{l}\text { Data are shown as } \\
\text { control rats; }{ }^{2} p<0\end{array}$ & 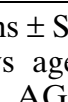 & 001 & $\begin{array}{l}\left(\mathrm{N}^{\mathrm{G}} \text {-nitro-L-ars }\right. \\
\text { globin }){ }^{3} \text { mean }\end{array}$ & $\begin{array}{l}\text { methyl ester); } \mathrm{HbA}_{10} \\
\text { veeks } 8-32 \text { for study } 1 \mathrm{C}\end{array}$ & cated haemo- \\
\hline
\end{tabular}

NADPHd histochemistry where $\beta$-NADPH was omitted from the reaction solution, staining also did not occur.

Statistical analysis. NADPHd-positive cells in all retinas were counted by eye using a projectorscope (Olympus BH-2) in a double blinded manner. The number of NADPHd-positive cells per retina was assessed by counting the number of cells within or touching the upper and right borders of a grid, the area of which was equivalent to $0.45 \mathrm{~mm}^{2}$ at $300 \times$ magnification. Counts were made at $2 \mathrm{~mm}$ intervals by scanning systematically in a roster fashion across the entire retina. From the total number of cells counted in the area of retina examined, the average density of NADPHd-positive neurons per $\mathrm{mm}^{2}$ of retina was determined. The total area of the retina was determined from a projected image of the whole-mount retinas using a digitizing pad interfaced to a computer. From this figure and the average density per retina the total number of NAD$\mathrm{PHd}$-positive neurons per retina was calculated.

To determine whether there is a difference in vessel density in experimental diabetes, point counting of retinal vessels in control $(n=7)$ and diabetic $(n=7)$ rats from the long-term group was done. Retinas were projected as above at $150 \times$ magnification and a $10 \times 10 \mathrm{~cm}$ grid was randomly placed over the projected image. The number of vessels touching intersecting points of the grid was assessed and expressed as a percentage of the total number of available points.

All values are expressed as means \pm SEM. Comparison of the mean values among the groups was done by analysis of variance (ANOVA). Comparisons between groups were done by Fisher's least significant difference method. In general a $p$ value less than 0.05 was considered to be significant, but in some studies where there were multiple comparisons a $p$-value less than 0.01 was used to assess statistical significance.

\section{Results}

Serial body weights and serum glucose for all study groups are shown in Table 1 (study 1; A: short term 1 week; B: short term 3 weeks; C: study long term, 32 weeks and study 2; D: 3 weeks NN0028). As observed previously diabetic rats had reduced body weights and markedly increased plasma glucose concentrations and glycated haemoglobin $\left(\mathrm{HbA}_{1 \mathrm{c}}\right)$ concentrations compared with control rats. Rats in the euglycaemic group had lower blood glucose concentrations than rats in other diabetic groups. Diabetes was also associated with reduced weight gain over the study period. In diabetic rats treated with aminoguanidine, L-NAME or NN0028, body weight and serum glucose concentrations were not different from untreated diabetic animals at 3 or 32 weeks.

Systolic blood pressure is shown in Tables 1A-D. In long term diabetic animals this variable was calculated as a mean of the data obtained from 8-32 weeks. Diabetic rats had a modest increase in blood pressure throughout the duration of the study compared with controls rats. At 3 weeks, aminoguanidine prevented the increase in systolic blood pressure in the diabetic rats (Table 1B). At 32 weeks, systolic blood pressure remained elevated in diabetic animals treated with either aminoguanidine or LNAME. 

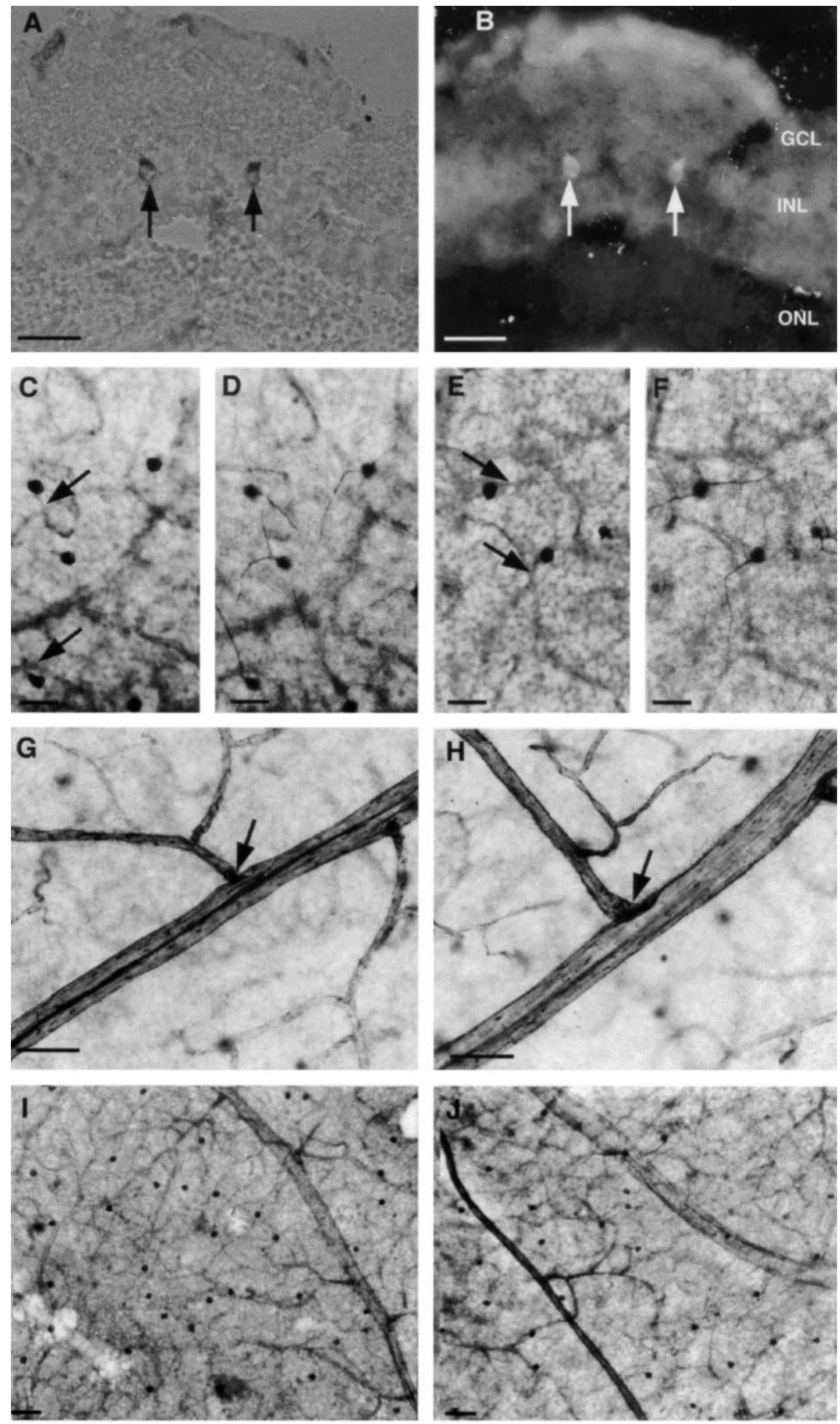

Fig. 1. NADPHd histochemistry (A) and nNOS-IR (B) are colocalised in amacrine cells (arrows) in the inner nuclear layer (INL) of the 3 week diabetic rat retina. These neurons are seen to be positioned in close proximity to the retinal vasculature $(\mathbf{C})$. The intensity of staining, and morphology in the 32 week diabetic retina [E (plane of focus at cell bodies in INL) and $\mathbf{F}$ (plane of focus on cell processes in inner plexiform layer (IPL)] is equal to the age matched control retina [ $\mathbf{C}$ (cell

bodies, INL) and $\mathbf{D}$ (cell processes, IPL)]. Staining of the retinal vasculature in both control $(\mathbf{G})$ and 32 weeks diabetic $(\mathbf{H})$ retinas is of equal intensity; note intense staining of precapillary sphincters (arrows). Low power views of retinal whole mounts in 32 week control (I) and diabetic (J) retinas shows that the retinal vasculature is well stained by NADPHd histochemistry and that there is a reduction in NADPHd-positive neurons after 32 weeks of diabetes. (Scale Bar $=50 \mu \mathrm{m} \mathbf{A}-\mathbf{J}$ ) 
NADPHd and nNOS-IR co-localisation. In untreated diabetic animals, it was shown that nNOS-IR and NADPHd histochemistry were $100 \%$ co-localized in amacrine cells in the inner nuclear layer (INL). An example of this is shown in Figures 1A (NADPHd histochemistry) and 1B (nNOS-IR). Of the total of 70 cells examined in transverse cryostat sections, all stained positively for NADPHd and nNOS-IR. There was no NADPHd-positive neuron that did not show nNOS-IR or vice versa.

NADPHd histochemistry. NADPHd histochemistry in the rat retina stained one class of amacrine cell which is located in the inner nuclear layer or occasionally displaced to the ganglion cell layer and closely related to the vasculature (Fig. $1 \mathrm{C}$ and E). There was no qualitative difference in the distribution, morphology or intensity of staining of NADPHd-positive amacrine cells in diabetic animals compared with control animals [Fig. 1C and D (control) and $1 \mathrm{E}$ and $\mathrm{F}$ (diabetic)]. Furthermore, there was no difference in the intensity of staining of the retinal vasculature [Fig. $1 \mathrm{G}$ (control) and $1 \mathrm{H}$ (diabetic)] and no qualitative difference in the appearance of the retinal vasculature after short or long term diabetes was observed. Point counting of the vessels in retinas from control $(n=7)$ and diabetic $(n=7)$ retinas at 32 weeks showed no difference in retinal vessel density. In both groups, $49 \%$ of points were in contact with a retinal vessel. In diabetic animals, no other retinal cells were reactive with NADPHd histochemistry. Of interest is the apparent intense NADPHd activity in the retinal arterioles, especially at the pre-capillary sphincters [Fig. $1 \mathrm{G}$ and $1 \mathrm{H}$ (arrows)]. This could relate to the action of the arterioles as major regulators of retinal capillary beds.

The effect of diabetes on the number of NADPHdpositive neurons. Diabetes resulted in a $32 \%$ reduction in the number of NADPHd-positive neurons in the retinas of diabetic animals compared with control rats after 1 week of STZ induced diabetes [2659 \pm 58 (control) vs $1816 \pm 85$ cells/retina (diabetic) $(p<0.05)]$. A similar reduction in the number of NADPHd-positive neurons was observed in diabetic rats after 3 weeks (Fig.2A) or 32 weeks (Fig.2B) compared with age-matched control rats. Examples of control and diabetic retinas 3 weeks after induction of diabetes are shown in Figure 1I (control) and $1 \mathrm{~J}$ (diabetic). In the group of diabetic rats rendered euglycaemic for 3 weeks there was no statistically significant difference in the number of NADPHd-positive neurons per retina when compared with the age matched control group (Fig. 2A).

After 3 weeks of treatment with aminoguanidine the number of NADPHd-positive neurons in the diabetic rat retina was not considerably different from control levels (Fig. 2A). This was also observed in diabetic rat retinas after 32 weeks of treatment with
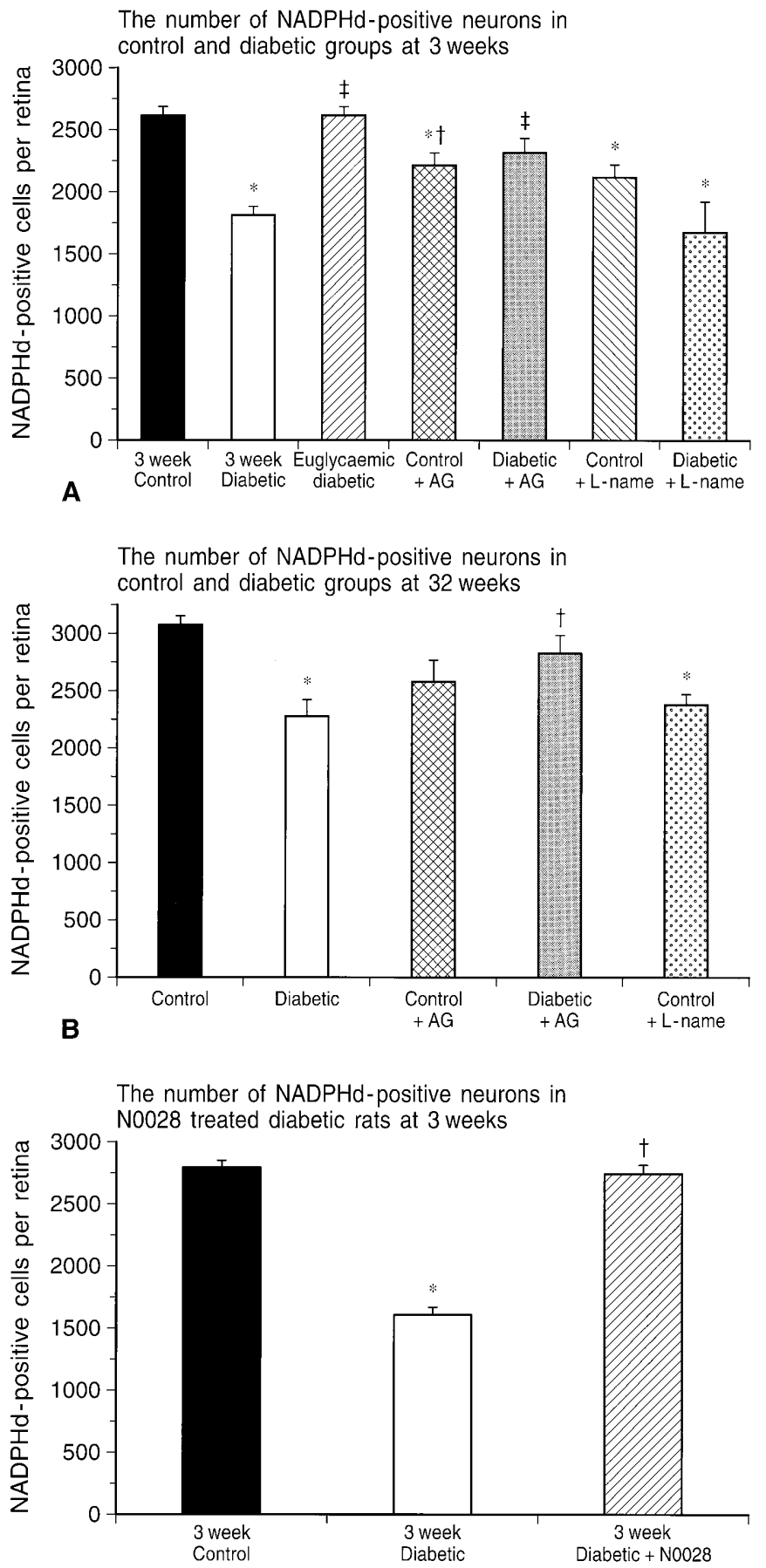

Fig. 2. Data are shown as means \pm SEM for number of NADPHd-positive neurons per retina; analysis was done by ANOVA. (A.) The effect of insulin, aminoguanidine (AG) and LNAME treatment in control and diabetic rats at 3 weeks; $* p<0.01$ vs age-matched control rats; $\ddagger p<0.05, \dagger p<0.01$ vs untreated diabetic rats $(\mathrm{F}=8.64, p<0.001)$. (B.) The effect of aminoguanidine (AG), and L-NAME treatment in control and diabetic groups after 32 weeks, $* p<0.01$ vs age-matched control rats; $\uparrow p<0.01$ vs untreated diabetic rats $(\mathrm{F}=4.37$, $p=0.007)$. (C.) The effect of NN0028 treatment in diabetic rats after 3 weeks, $* p<0.01$ vs age-matched control rats; $\dagger p<0.01$ untreated diabetic rats $(\mathrm{F}=104.8, p<0.001)$ 
aminoguanidine (Fig. 2B). L-NAME-treatment in diabetic animals showed no evidence of preventing the diabetes-associated depletion in the number of NADPHd-positive neurons at 3 or 32 weeks of treatment, the number of NADPHd-positive neurons remaining well below control levels (Fig.2A and B). Aminoguanidine and L-NAME treatment in control groups resulted in a considerable reduction in the total number of NADPHd-positive neurons. To assess the effect of NN0028 in this study, the number of NADPHd-positive amacrine cells in the untreated diabetic group was markedly reduced compared with control animals, while NN0028 treatment prevented this depletion (Fig. 2C).

\section{Discussion}

This study focused on the effects of STZ-induced diabetes on the population of nNOS-containing amacrine cells in the rat retina. The enzyme NOS exists in three isoforms: two constitutive forms; neuronal (nNOS), endothelial (eNOS) and one inducible form (iNOS). All three isoforms of NOS have been implicated in the pathogenesis of diabetic retinopathy [11]. In the cerebral cortex, NO released from nNOScontaining neurons has been shown to directly regulate cerebral blood flow [4]. The retina lacks an adrenergic innervation of its vasculature, and nNOS localised in amacrine cells closely related to the retinal vasculature [3] might be of relevance to the alteration in the autoregulation of retinal blood flow which has been reported to occur in diabetes [12]. Since NADPHd and nNOS are $100 \%$ co-localised in the diabetic and control rat retina, we have used NADPHd histochemistry in this study to investigate the effect of diabetes on nNOS-containing neurons in the retina.

We have shown a reduction of nNOS containing neurons in the diabetic rat retina after 1 and 3 weeks of STZ-induced diabetes; this reduction persists at 32 weeks of experimental diabetes. It could be argued that the alteration of nNOS is a consequence of direct STZ toxicity. The reduction in the number of nNOS-containing retinal amacrine cells shown in this study, however, was not transient, but consistently detected throughout the observation period. Furthermore, in the group of animals made euglycaemic for 3 weeks after induction of diabetes, there was no relevant reduction in the number of nNOS-containing amacrine cells compared with their age-matched control animals.

The results of this investigation support the findings of other investigators suggesting that NOS activity is reduced in diabetes. For example, NO deficiency has been implicated in the slowing of nerve conduction [13] and in decreased vascular relaxation [14] in the acutely STZ-diabetic rat while others have reported NO deficiency of cells in cultures exposed to hyper- glycaemia [15]. We suggest that a loss of nNOS activity might contribute to retinal neurovascular dysfunction in experimental diabetes. One must be cautious, however, in assuming that reduced nNOS immunoreactivity correlates directly with reduced nNOS activity.

In this study no quantitative measure of other NOS isoforms was made. It has been reported that the expression of iNOS may be up-regulated in the Müller glial cells, endothelial cells and pericytes of the retina under certain conditions [2] and that diabetic complications could be mediated by a relative or absolute increase in NOS activity [11]. Since NADPH diaphorase can detect all isoforms of NOS, however, and in the diabetic animals there was no NADPH diaphorase staining in cell populations other than those seen in control animals, we have no evidence of diabetes inducing the expression of NOS.

Advanced glycation end products (AGEs) have been clearly implicated in the genesis of diabetic retinopathy with evidence in long term diabetic rats that aminoguanidine prevents retinal AGE accumulation and early structural markers of retinopathy [8]. The early appearance of AGEs has been shown in the vasculature of isolated mesenteric arterioles in STZ-induced diabetes [16]. The accumulation of AGEs in these sites is inhibited by aminoguanidine treatment. Similar results have been found in studies on retinal AGE concentrations using techniques such as autofluorescence of retinal digests [5] and confirmed in our laboratory using immunohistochemistry [8].

While the results of both in vivo and in vitro experiments suggest that aminoguanidine is retinoprotective [5], conflicting views exist as to whether this effect is mediated through inhibition of advanced glycation or NOS or both. The prevention of AGE formation by aminoguanidine has been attributed largely to its ability to form stable complexes with reactive dicarbonyls (e.g. 3-deoxyglucosone) and aldehydes produced by non-enzymatic glycation reactions [17] and lipid peroxidation. Furthermore, it has been suggested that aminoguanidine also scavenges reactive products (Schiff bases and Amadori products), thereby preventing damage to cellular and extracellular constituents [18]. It has been shown in the STZ-induced diabetic rat that aminoguanidine rapidly reverses slowing of nerve conduction velocity and blood flow via improved NO action and that this effect is likely to be mediated through aminoguanidine's action of inhibition of free radical production by autoxidative glycosylation or glycoxidation [13]. In addition, aminoguanidine has also been shown to be a potent inhibitor of iNOS and of nNOS to a lesser extent [11]. Both aminoguanidine and L-NAME (a nonselective inhibitor of constitutive and inducible isoforms of NOS) prevent vascular dysfunction in diabetic rats and non-diabetic rats [19]. Furthermore, both compounds attenuate pathophysiological and structural changes that are linked to the increased ac- 
tivity of iNOS in immune- and endotoxin-mediated diseases in non-diabetic animals [20].

Therefore, we were interested in determining whether aminoguanidine treatment can prevent the diabetes-induced depletion of nNOS-containing amacrine cells, and if so whether this effect was mediated via the inhibition of AGE accumulation or by inhibition of NOS isoforms. The ability of NN0028, an inhibitor of AGE formation, but not NO formation and the failure of L-NAME to prevent the depletion of nNOS-containing neurons in the diabetic retina, is consistent with the hypothesis that the effect of aminoguanidine is primarily the suppression of AGEformation and accumulation. The observation that aminoguanidine reduces the number of nNOS-containing neurons in control retinas might suggest that this agent has differential effects in normal and diabetic retinas; in normal retinas it is possible that its action as an inhibitor of NOS predominates. This issue requires further examination including assessment of other NOS isoforms.

It is likely that other mechanisms not examined in this study such as the polyol pathway-dependent reduction in NOS activity [21] or pathways linking Dglucose to diacylglycerols (DAGs) and protein kinase $\mathrm{C}$ (PKC) [22] are also involved in the regulation of NOS. The restoration of the number of nNOS-containing neurons to control levels with NN0028 and aminoguanidine treatment, however, suggests that advanced glycation is an important contributor to the observed changes.

This study has clearly documented changes in nNOS in the retina after STZ-induced diabetes. These changes possibly have a functional relevance to the development of diabetic retinopathy since the NO produced in nNOS-containing cells is a potent regulator of vascular tone in the cerebral cortex [4] and might play a similar role in the retina. Retinal haemodynamics have been postulated to play an important role in the genesis and progression of diabetic retinopathy [1]. It is our prediction that a decrease in nNOS-containing amacrine cells would result in a decrease in retinal blood flow as has been reported in diabetes by some authors [23]. Other studies [11], however, report an increase in retinal blood flow in diabetes hence the loss of nNOS-containing retinal neurons might be more relevant to a disturbance in the autoregulatory capacity of the retinal circulation for which there is more general agreement [12].

Acknowledgements. This work was supported by a grant from Juvenile Diabetes Foundation International.

\section{References}

1. Kohner EM, Patel V, Rassam SM (1995) Role of blood flow and impaired autoregulation in the pathogenesis of diabetic retinopathy. Diabetes 44: 603-607
2. Goldstein IM, Ostwald P, Roth S (1996) Nitric oxide: a review of its role in retinal function and disease. Vision Res 36: 2979-2994

3. Roufail E, Stringer M, Rees S (1995) Nitric oxide synthase immunoreactivity and NADPH diaphorase staining are co-localised in neurons closely associated with the vasculature in rat and human retina. Brain Res 684: 36-46

4. Cholet N, Seylaz J, Lacombe P, Bonvento G (1997) Local uncoupling of the cerebrovascular and metabolic responses to somatosensory stimulation after neuronal nitric oxide synthase inhibition. $\mathbf{J}$ Cereb Blood Flow Metab 17: 1191-1201

5. Hammes HP, Martin S, Federlin K, Geisen K, Brownlee M (1991) Aminoguanidine treatment inhibits the development of experimental diabetic retinopathy. Proc Natl Acad Sci USA 88: $11555-11558$

6. Nathan C (1997) Nitric Oxide as a secretory product in mammalian cells. FASEB J 6: 3051-3064

7. Cooper ME, Rumble J, Komers R, Du HC, Jandeleit K, Chou ST (1994) Diabetes-associated mesenteric vascular hypertrophy is attenuated by angiotensin-converting enzyme inhibition. Diabetes 43: $1221-1228$

8. Soulis T, Thallas V, Youssef S et al. (1997) Advanced glycation end products and their receptors co-localise in rat organs susceptible to diabetic microvascular injury. Diabetologia 40: 619-628

9. Schmidt FH (1961) Enzymatic determination of glucose and fructose simultaneously. Klin Wochenschr 39: 1244-1247

10. Bunag RD (1973) Validation in awake rats of a tail-cuff method for measuring systolic pressure. J Appl Physiol 34: 279-282

11. Tilton RG, Chang K, Hasan KS et al. (1993) Prevention of diabetic vascular dysfunction by guanidines. Inhibition of nitric oxide synthase versus advanced glycation end-product formation. Diabetes 42: $221-232$

12. Cringle SJ, Yu DY, Alder VA, Su EN (1993) Retinal blood flow by hydrogen clearance polarography in the streptozotocin-induced diabetic rat. Invest Ophthalmol Vis Sci 34: 1716-1721

13. Cameron NE, Cotter MA (1996) Rapid reversal by aminoguanidine of the neurovascular effects of diabetes in rats: modulation by nitric oxide synthase inhibition. Metabolism 45: 1147-1152

14. Archibald V, Cotter MA, Keegan A, Cameron NE (1996) Contraction and relaxation of aortas from diabetic rats: effects of chronic anti-oxidant and aminoguanidine treatments. Naunyn Schmiedebergs Arch Pharmacol 353: 584-591

15. Shindo H, Thomas TP, Larkin DD et al. (1996) Modulation of basal nitric oxide-dependent cyclic-GMP production by ambient glucose, myo-inositol, and protein kinase C in SH-SY5Y human neuroblastoma cells. J Clin Invest 97: 736-745

16. Rumble JR, Cooper ME, Soulis T et al. (1997) Vascular hypertrophy in experimental diabetes. Role of advanced glycation end products. J Clin Invest 99: 1016-1027

17. Fu MX, Wells Knecht KJ, Blackledge JA, Lyons TJ, Thorpe SR, Baynes JW (1994) Glycation, glycoxidation, and cross-linking of collagen by glucose. Kinetics, mechanisms, and inhibition of late stages of the Maillard reaction. Diabetes 43: 676-683

18. Nyengaard JR, Chang K, Berhorst S, Reiser KM, Williamson JR, Tilton RG (1997) Discordant effects of guanidines on renal structure and function and on regional vascular dysfunction and collagen changes in diabetic rats. Diabetes 46: 94-106

19. Corbett JA, Tilton RG, Chang K et al. (1992) Aminoguanidine, a novel inhibitor of nitric oxide formation, prevents diabetic vascular dysfunction. Diabetes 41: 552-556

20. Cross AH, Misko TP, Lin RF, Hickey WF, Trotter JL, Tilton RG (1994) Aminoguanidine, an inhibitor of inducible nitric oxide synthase, ameliorates experimental autoimmune encephalomyelitis in SJL mice. J Clin Invest 93: 2684-2690

21. Cameron NE, Cotter MA (1993) Contraction and relaxation of aortas from galactosaemic rats and the effects of aldose reductase inhibition. Eur J Pharmacol 243: 47-53

22. Craven PA, Studer RK, DeRubertis FR (1994) Impaired nitric oxide-dependent cyclic guanosine monophosphate generation in glomeruli from diabetic rats. Evidence for protein kinase C-mediated suppression of the cholinergic response. J Clin Invest 93: 311-320

23. Bursell SE, Clermont AC, Shiba T, King GL (1992) Evaluating retinal circulation using video fluorescein angiography in control and diabetic rats. Curr Eye Res 11: 287-295 